\title{
Next generation sequencing for diagnosis of central nervous system aspergillosis in liver transplant recipients
}

\author{
Fang Chen ${ }^{1,2}$, Yujing Zhao ${ }^{3}$, Chuan Shen ${ }^{1}$, Longzhi Han ${ }^{1}$, Xiaosong Chen ${ }^{1}$, Jianjun Zhang ${ }^{1}$, Qiang Xia ${ }^{1}$, \\ Yongbing Qian ${ }^{1 \wedge}$ \\ ${ }^{1}$ Department of Liver Surgery, Renji Hospital, School of Medicine, Shanghai Jiaotong University, Shanghai, China; ${ }^{2}$ Department of Pharmacy, Renji \\ Hospital, School of Medicine, Shanghai Jiaotong University, Shanghai, China; ${ }^{3}$ Department of Anesthesiology, Second Xiangya Hospital, Central \\ South University, Changsha, China \\ Contributions: (I) Conception and design: F Chen, Y Qian; (II) Administrative support: J Zhang, Q Xia; (III) Provision of study materials or patients: \\ Y Zhao, C Shen, L Han; (IV) Collection and assembly of data: Y Zhao; (V) Data analysis and interpretation: F Chen, Y Qian; (VI) Manuscript \\ writing: All authors; (VII) Final approval of manuscript: All authors. \\ Correspondence to: Yongbing Qian. Department of Liver Surgery, Renji Hospital, School of Medicine, Shanghai Jiaotong University, Shanghai, China. \\ Email: qianyb79@hotmail.com.
}

Background: Fungal encephalitis is uncommon and sometimes fatal in liver transplant (LT) recipients. Early diagnosis of central nervous system (CNS) fungal infections, especially aspergillosis, is difficult based on routine tests of cerebrospinal fluid (CSF) alone. Next-generation sequencing (NGS) as a new tool may help in this respect.

Methods: Shotgun metagenomics was used to detect pathogens in CSF of patients, who were clinically suspected of CNS infection. Sequencing was performed at BGIseq-50 platform (BGI, Shenzhen).

Results: NGS technique identified Aspergillus in CSF of 5 patients, who were suspected of CNS infection, although clinical symptoms of these patients varied dramatically. The resulting sequence reads corresponding to Aspergillus species ranged from 2 to 25 , with genomic coverage ranging from $0.0003 \%$ to $0.0036 \%$. Rapid identification of Aspergillus enabled early appropriate antifungal therapy, although 4 patients eventually died of severe infection.

Conclusions: This is the first study to highlight the utility of NGS in early diagnosis of CNS aspergillosis in LT recipients. This new tool may be helpful in improving the diagnosis of CNS aspergillosis.

Keywords: Aspergillus; central nervous system (CNS); cerebrospinal fluid; liver transplantation; next generation sequencing

Submitted Jan 07, 2021. Accepted for publication Apr 08, 2021.

doi: 10.21037/atm-21-92

View this article at: http://dx.doi.org/10.21037/atm-21-92

\section{Introduction}

Secondary central nervous system (CNS) infection is the second most common neurological complication next to cerebrovascular accidents, accounting for $20-25 \%$ of all CNS lesions after liver transplantation (LT) (1). Liver transplant recipients have the higher rate of disseminated disease with invasive mold infection than other SOT. CNS infection primarily occurred in the first 3-4 weeks after LT. Previous studies have estimated that the mortality was $64 \%$ in LT recipients complicated with invasive aspergillosis, while the mortality rates were $82 \%$ and $100 \%$, respectively, in patients who developed invasive aspergillosis after retransplantation and in those undergoing re-transplantation more than 30 days after primary transplantation $(2,3)$, because of limited surgical options, poor drug penetration

\footnotetext{
^ ORCID: 0000-0003-4081-428X.
} 
through the blood brain barrier, antifungal resistance, and poor tolerance to antifungal agents (4). However, the clinical manifestations of CNS infections, even lifethreatening, may be subtle and difficult to diagnose.

Next-generation sequencing (NGS) is a new technique, which is increasingly used in the identification of challenging CNS pathogens (5-7). In this study, we showed the potential of NGS in identifying CNS aspergillosis in LT recipients for appropriate antifungal therapy.

We present the following article in accordance with the MDAR reporting checklist (available at http://dx.doi. org/10.21037/atm-21-92).

\section{Methods}

\section{Patients}

During the study period, the total 800 of adult liver transplantation were finished in our institution, including 794 deceased donor and 6 living donor liver transplantation.

Five LT recipients who were clinically suspected of CNS fungal infection were included in this study. These patients were admitted to Renji Hospital from July 2017 to December 2019. The demographic, clinical, radiological, etiological, and treatment outcome data were retrieved from hospital information system.

The study was conducted in accordance with the Declaration of Helsinki (as revised in 2013). The protocol was reviewed and approved by the institutional review committee of Renji Hospital (approval No: 2018-136) and individual consent for this retrospective analysis was waived. All the organs transplanted to the patients were donated voluntarily. No donor organs were obtained from executed prisoners.

Standard IS regimens include high-dose prednisone and basiliximab induction, followed by tacrolimus, mycophenolic acid, and prednisone in our center. The target serum level of tacrolimus was $8-12 \mathrm{ng} / \mathrm{mL}$ during the first month of LT and 6-8 ng/mL during the first 6 mo of LT. The target serum level of cyclosporin was $150-200 \mathrm{ng} / \mathrm{mL}$ during the first month and $120-150 \mathrm{ng} / \mathrm{mL}$ in the first 6 mo of LT. When severe infection was suspected, decrease dosage of prednisone and/or delayed CNI were employed.

\section{Metagenomic next-generation sequencing and analysis}

About $0.5-3 \mathrm{~mL}$ CSF sample was collected from each patient. The CSF sample $(0.5 \mathrm{~mL})$ was mixed with $1 \mathrm{~g}$
$0.5 \mathrm{~mm}$ glass beads (Sigma-Aldrich, USA) in a $1.5 \mathrm{~mL}$ microcentrifuge tube. The tubes were agitated vigorously at $1,500 \mathrm{~g}$ for $30 \mathrm{~min}$ on VORTEX-GENIE 2 VORTEX MIXER 12 (Scientific Industries, USA). Then $0.3 \mathrm{~mL}$ of the specimen was separated into a new $1.5 \mathrm{~mL}$ microcentrifuge tube. Total DNA was extracted using the TIANamp Micro DNA Kit (DP316, TIANGEN BIOTECH) according to the manufacturer's instruction. The total DNA were fragmented by Bioruptor ${ }^{\circledR}$ Pico (Diagenode, Belgium) to $150 \mathrm{bp}$. After fragmentation, poly A tail was added to the fragmented sequence for end repair purpose. Samplespecific adapter was added to the DNA sequences, and unbiased PCR was conducted for sequence amplification. DNA libraries were purification with magnetic beads purification strategy using MGIEasy DNA purification kit (MGI, China) after PCR amplification. The quality of the resulting library was measured by Qubit Fluorometer 3.0 (Invitrogen, USA) and Agilent 2100 Bioanalyzer (Agilent Technologies, USA). Qualified libraries ( $>2 \mathrm{ng} / \mathrm{uL}$, medium sequence size between 200-300 bp) were sequenced on BGISEQ-50 system (8). At least 20 million reads were produced from each sample.

\section{Data processing and analysis}

After removing low-quality, and short (length $<35 \mathrm{bp}$ ) reads, and computational subtraction of human host sequences mapped to the human reference genome (hg19) using Burrows-Wheeler Alignment tool (version 0.7.10r789), at least 20 million high-quality unique reads were finally generated from each sample (9). After removal of low-complexity reads using prinseq tool (version 0.20.3), the remaining data were classified by simultaneously aligning to locally built reference databases, Microbial Genome Databases. The reference databases for microbial classification downloaded from NCBI containing 4,061 whole genome sequences of viral taxa, 2,473 bacterial genomes or scaffolds, 199 fungi and 135 parasites that are associated with human diseases. Data Analytics algorithms were used to exclude the microorganisms that were not significantly related to infection. Genus/species-specific reads uniquely aligned to infection-related microorganisms were reported.

\section{Statistical analysis}

Comparative analysis were evaluated with the Pearson $\chi^{2}$ or the Fisher exact test when necessary. Variables with a $\mathrm{P}$ value 
Table 1 Clinical features of 5 liver transplant recipients complicated with central nervous system aspergillosis

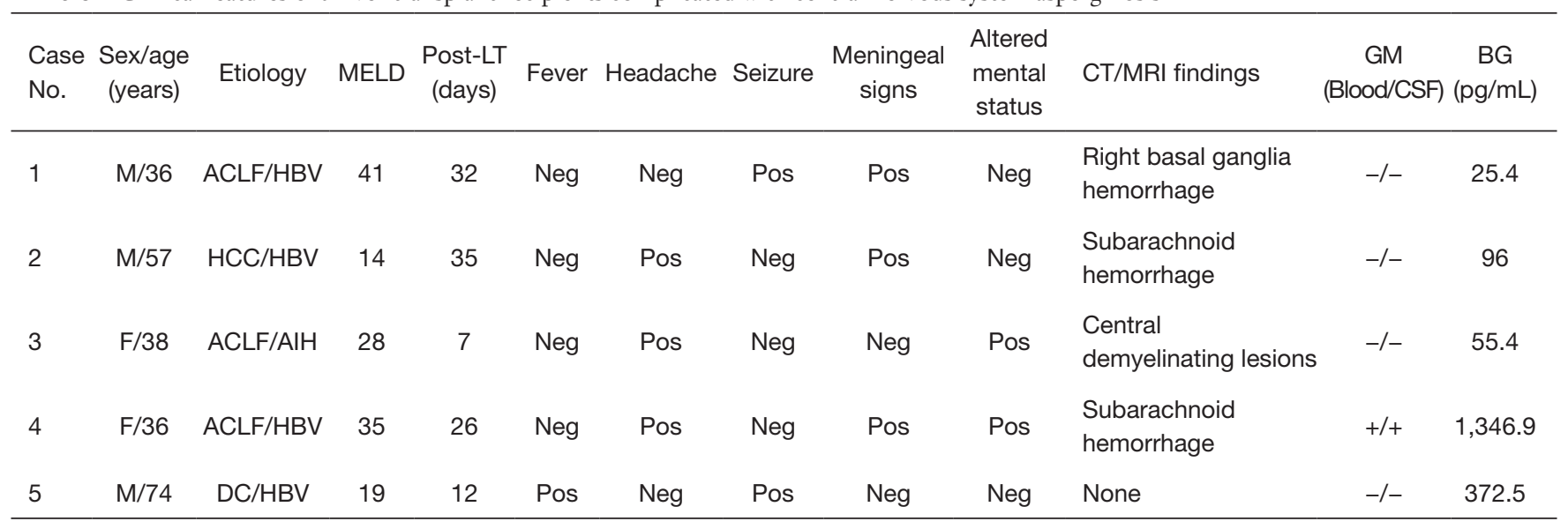

$\mathrm{AlH}$, autoimmune hepatitis; ACLF, acute-on-chronic liver failure; DC, decompensated cirrhosis; CT, computed tomography; HBV, hepatitis B virus; HCC, hepatocellular cancer; LT, liver transplant; MRI, magnetic resonance imaging; GM, Galactomannan (Positive: index $\geq 0.65$ ); BG, $\beta$-Dglucan; Neg, negative; Pos, positive.

$<0.05$ (two-tailed) were considered statistical significant. All statistical analyses were performed by the SPSS20.0.

\section{Results}

\section{Clinical characteristics of patients}

Five patients were identified in a cohort of patients with clinically suspected CNS aspergillosis, including 3 males and 2 females, aged from 36 to 74 years. The onset time of infection was 7 to 35 days after LT. Five patients with high MELD score, from 14 to 41 , Three of five patients were diagnosed with acute-on-chronic liver failure, 1 was $\mathrm{HBV}$-related hepatocellular cancer, 1 was HBV-related decompensated cirrhosis. The clinical manifestations of these patients are summarized in Table 1. The most common prodromal symptoms were vertigo, nausea, seizures, fever, and headache. Three patients experienced intracranial hemorrhage and two progressed rapidly to coma. Contrast brain magnetic resonance imaging (MRI) was performed in all patients. Contrast-enhanced T1-weighted image showed nodular and circular enhancement in two cases. T2-weighed imaging, and fluid-attenuated inversion recovery (FLAIR) imaging detected multiple abscesses with surrounding edema suggestive of intracranial hemorrhage. Diffusion-weighted imaging (DWI) showed limited diffusion of the lesion and high signal (Figure 1).

\section{Routine and biochemical CSF tests}

Lumbar punctures revealed increased opening pressure (up to $260 \mathrm{mmH}_{2} \mathrm{O}$ ) of the CSF in cases 1 and 3 . CSF panel tests showed elevated white blood cell count up to $320 \times 10^{6} / \mathrm{L}$; increased level of protein (up to three times of normal value); decreased glucose level in only 1 case; and normal chloride level. CSF culture of bacteria and fungi revealed negative results. Patient 4 had positive outcome of galactomannan antigens in both CSF and serum, patient 4 and 5 had elevated beta-glucan in serum, the remaining were negative.

These patients were also complicated with lung, bloodstream, or catheter-related infections due to bacteria. In this study, patient 2 and 4 had probable invasive pulmonary aspergillus prior to CNS involved IA infection, patient 3 had bloodstream infection of Cryptococcus neoformans and pulmonary infection of Klebsiella pneumoniae, patient 1 and 5 had pulmonary infection of Acinetobacter baumannii and bloodstream infection of Propionibacterium acnes, respectively (Table 2).

\section{NGS results of CSF}

Aspergillus species-specific DNA was detected in CSF of all 5 patients. The number of raw reads ranged from 29385028 to 34381986 . The sequence reads corresponding to Aspergillus species ranged from 2 to 25 . Genomic coverage ranged from $0.0003 \%$ to $0.0036 \%$. Table 3 presents the details of the NGS results of CSF. The coverage and depth of each species were calculated and ranked from high to low based on coverage and unique reads. Aspergillus sydowii was identified in 3 cases. Aspergillus fumigatus was identified in 

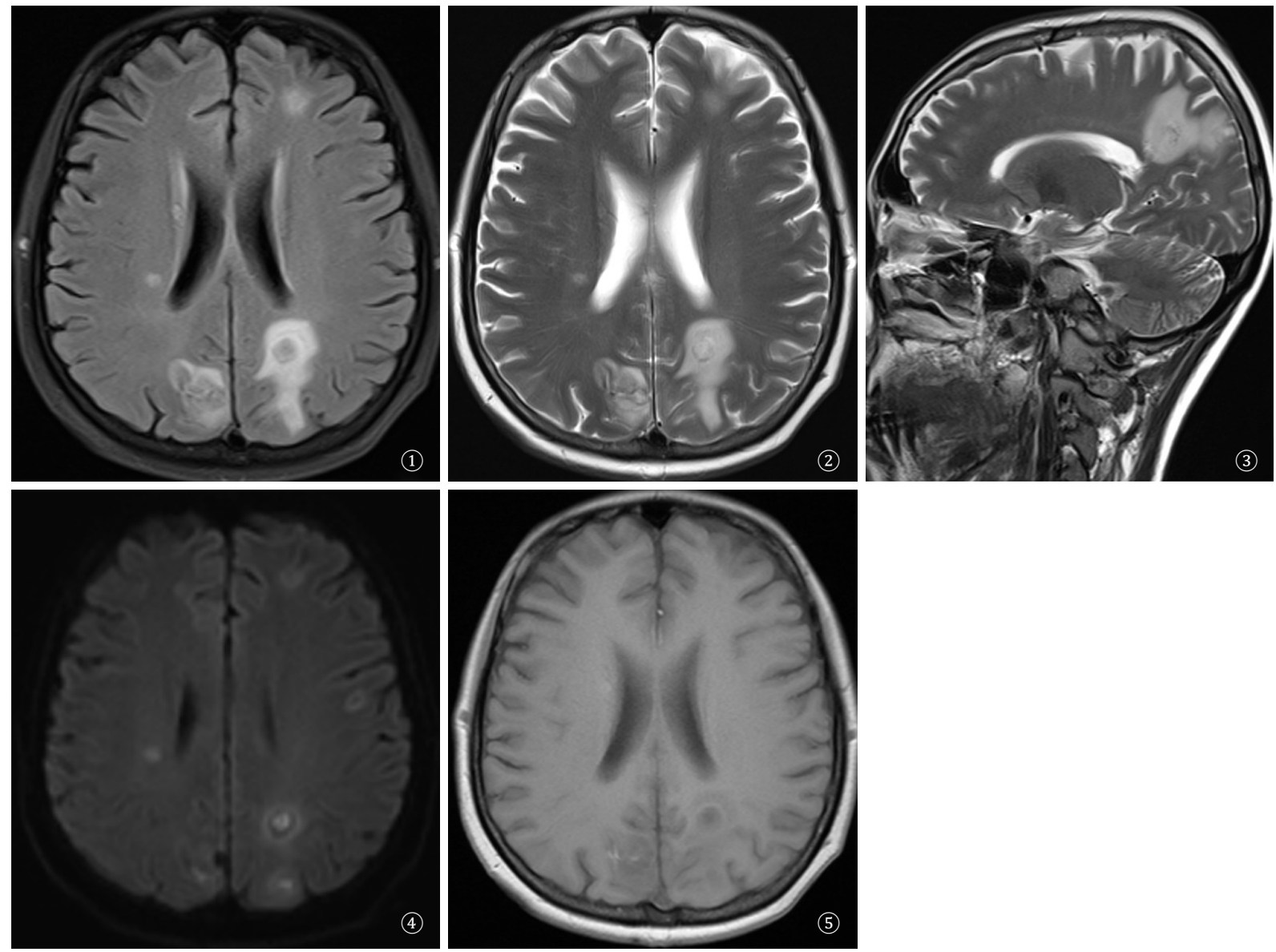

Figure 1 Magnetic resonance imaging revealed the central nervous system lesions in 1 liver transplant recipient. Panels 1 to 3: T2 Flair and T2WI showed multiple abscesses with surrounding edema, suggestive of intracranial hemorrhage in white matter of bilateral frontal and parietal lobe. Panel 4: DWI showed limited diffusion of the lesion and high signal within bilateral frontal and parietal lobe. Panel 5: T1WI enhanced scan showed nodular and circular enhancement within bilateral frontal and parietal lobe.

Table 2 Routine laboratory tests of cerebrospinal fluid in five liver transplant recipients

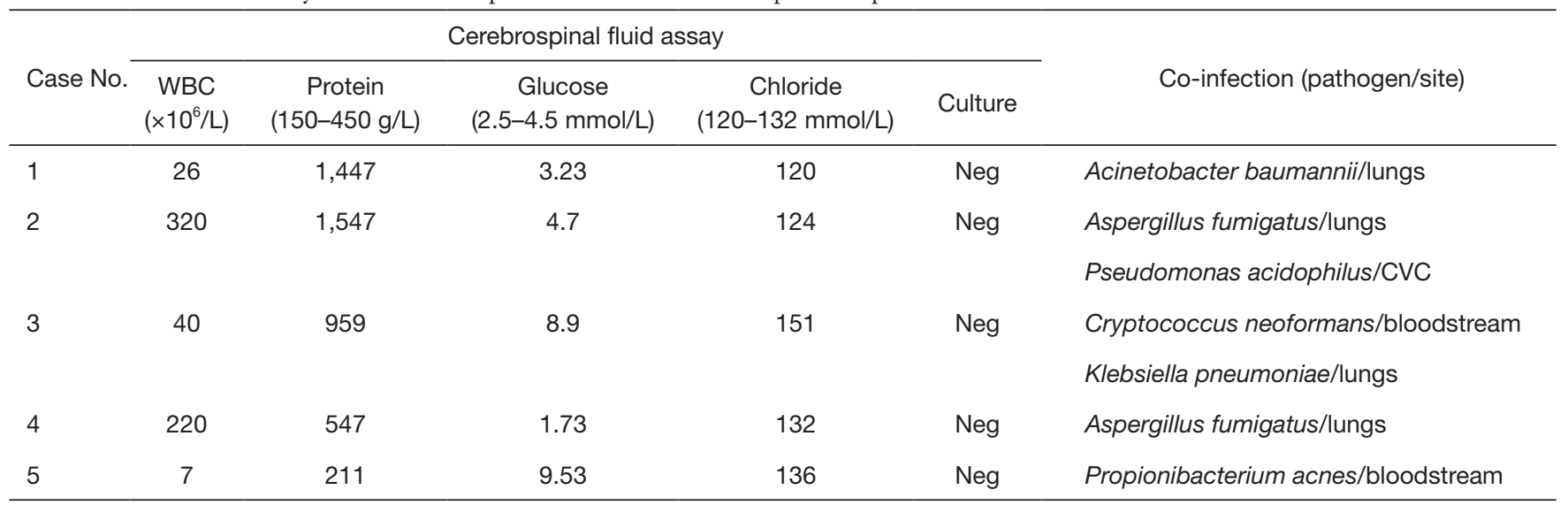

CVC, central venous catheter; Neg, negative; WBC, white blood cell. 
Table 3 Next-generation sequencing of cerebrospinal fluid for Aspergillus species in 5 patients

\begin{tabular}{lcccc}
\hline Case No. & Pathogen identified & No. of unique reads & Coverage, \% & Depth \\
\hline 1 & Aspergillus sydowii & 8 & 0.0012 & 1.0 \\
2 & Aspergillus fumigatus & 9 & 0.0015 & 1.0 \\
3 & Aspergillus sydowii & 3 & 0.0004 & 1.0 \\
4 & Aspergillus fumigatus & 2 & 0.0003 & 1.0 \\
5 & Aspergillus sydowii & 25 & 0.0036 & 1.0 \\
\hline
\end{tabular}

Table 4 Antimicrobial regimens before and after next-generation sequencing and clinical outcome

\begin{tabular}{llll}
\hline Case No. & Antimicrobial regimen before NGS & Antimicrobial regimen after NGS & Clinical outcome \\
\hline 1 & Moxifloxacin, Ceftriaxone & Cefoperazone/sulbactam, Voriconazole & Died \\
2 & Imipenem/cilastatin, Teicoplanin, Caspofungin & Imipenem/cilastatin, Voriconazole & Died \\
3 & Meropenem, Fluconazole & Ceftriaxone, Fluconazole, Voriconazole & Died \\
4 & Meropenem, Vancomycin, Voriconazole & Meropenem, Vancomycin, Voriconazole & Died \\
5 & Ceftazidime, Teicoplanin & Ceftriaxone, Teicoplanin, Voriconazole & Improved \\
\hline
\end{tabular}

NGS, next-generation sequencing.

2 cases. Cryptococcus was also revealed by NGS of CSF in patient 3.

Propionibacterium acnes, a common contaminating microorganism, was found by NGS present in all five patients. But Mycobacterium tuberculosis and Treponema pallidum were not detected by NGS of CSF.

\section{Clinical outcome}

NGS results did inform the change of antimicrobial therapy in all the five cases (Table 4). Voriconazole was therefore added to fight CNS aspergillosis. But, 3 of 5 developed with cerebral hemorrhage or Subarachnoid hemorrhage, 1 patient complicated with Central demyelinating lesions, 4 patients with cerebral severe complications died although timely voriconazole administration. Only one patient improved gradually after treatment.

\section{Discussion}

To our knowledge, this is the first case series study to examine the feasibility of NGS of CSF for the diagnosis of CNS aspergillosis in LT recipients. Five patients with clinically suspected CNS infection had dramatically different clinical presentations and unrevealing results of routine diagnostic workup for detecting etiology. NGS of CSF here provided useful information for identifying the potential pathogens of CSF infection.

CNS infection is a common and serious complication in patients after solid organ transplants due to long-term use of immunosuppressive agents (10), usually caused by bacteria, viruses, or fungi (11). Invasive candidiasis is the most common invasive fungal disease (56\%), followed by invasive aspergillosis (19\%) (12). Invasive aspergillosis is a rare but very fatal complication in LT recipients associated with poor outcome $(13,14)$. Previous studies have demonstrated that opportunistic CNS infection is increasingly associated with transplantation $(15,16)$. Invasive CNS aspergillosis is associated with higher mortality ( $88 \%$ to $99 \%$ ) than the invasive aspergillosis in other body sites such as lungs (17). Therefore, fast identification of CNS aspergillosis is crucial for timely and appropriate antifungal therapy, and therefore better patient outcome achieved.

Unfortunately, the diagnostic of CNS aspergillosis in LT patients is a considerable clinical challenge due to the following facts. Firstly, the clinical manifestations are usually non-specific due to absence of typical signs and symptoms of infection, and various non-infectious disease can also present similar characteristic as CNS infection. Secondly, some clinical manifestations of CNS aspergillosis, such as epilepsy, are similar to the adverse reactions of 
immunosuppressive agents (18). Thirdly, the probability of positive CSF culture is low due to low sensitivity of routine laboratory tests although positive culture is the gold standard for diagnosis. CNS aspergillosis is a complicated diagnosis, based on comprehensive modes, such as mycological, clinical and radiological analyzed together. Finally, conventional CSF tests are less sensitive to CNS aspergillosis. So, etiological diagnosis is difficult based on culture, serological identification, and specific pathogen PCR assay alone (6).

NGS is an emerging diagnostic technique for identifying potential pathogens of the CNS. Theoretically, almost all microbes can be uniquely identified based on specific nucleic acid sequences if there are long enough sequencing reads, high enough hits, and an all-inclusive reference database. Wilson and colleagues identified Leptospira santarosai in the CSF from a patient with meningoencephalitis by using unbiased NGS in 2014. From then on, there has been great interest in applying NGS technology to comprehensively identify infectious agents in CSF samples (19). The emerging case reports and clinical studies have demonstrated that NGS can be used as an effective tool for diagnosis of CNS infections $(6,20,21)$.

In the present study, unique reads corresponding to the Aspergillus genome were identified by NGS in the CSF of the five patients in a distinct amount, suggestive of Aspergillus sydowii in 3 cases and Aspergillus fumigatus in 2 cases. Furthermore, the NGS-based diagnosis of aspergillosis is consistent with the clinical symptoms, CSF, and neuroimaging evaluations. M. tuberculosis and T. pallidum were ruled out simultaneously in 4 patients as they were not detected by NGS of CSF. Cryptococcus neoformans was also detected in patient 3 . We adjusted the antimicrobial regimen based on the results of NGS of CSF. Voriconazole was added to treat aspergillosis in all patients. Fluconazole was also added in case 3 to target Cryptococcus neoformans.

The cryptic species Aspergillus sydowii was revealed by NGS in 3 cases with the unique reads ranging from 3 to 25 . The prevalence of cryptic species is $11 \%$ to $19 \%$ in LT recipients. Such species are indistinguishable from other species in terms of phenotypic characteristics and tend to be more resistant to the existing antifungal agents (22-24). Previous studies have reported that higher minimum inhibitory concentration (MIC) is usually required for antifungal agents to inhibit these cryptic species, for example, itraconazole MIC $6 \mathrm{mg} / \mathrm{L}$ for Aspergillus carneus; amphotericin B and itraconazole MIC of 4 and $16 \mathrm{mg} / \mathrm{L}$ respectively for Aspergillus sydowii (25). Aspergillus tubingensis is the most frequently identified cryptic species, accounting for $10.2 \%$ of all species and $81.5 \%$ of cryptic species (25). The proportion of other cryptic species such as $A$. sydowii (1.8\%), A. carneus (3.7\%), A. calidoustus (3.7\%), and $A$. tamari $(5.5 \%)$ is very low. However, these species were more frequently considered to be clinically relevant although their proportion is very low.

Contamination is a major issue in NGS $(26,27)$. Contaminating microorganisms such as $P$. acnes were also found in our study. It could be caused by crosscontamination between samples during preparation of the NGS library, contamination of the skin or normal flora during lumbar puncture, or incorrect sample separation or formation of artifact products $(28,29)$. The clinically relevant pathogens must be distinguished from contaminated microorganisms in NGS. Some common background microorganisms were also found in our NGS results. For example, there were relatively higher number of species-specific reads for $P$. acnes, Burkholderia, and Bradyrhizobia. Researchers therefore should have the knowledge of the common background microorganisms when interpreting the NGS results.

NGS of CSF is more useful in identifying the challenging and emerging CNS pathogens (e.g., Aspergillus) than conventional testing methods. Future clinical application of standardized NGS assay can screen all possible pathogens and result in better outcome for LT patients. NGS can also provide etiological evidence faster for patients with CNS aspergillosis, to initiate appropriate etiological treatment earlier, thus reduce mortality.

\section{Acknowledgments}

We thank Dr. Yang Zhou and Dr. Shengli Li in improving the manuscript, especially technical support and interpretation of mNGS.

Funding: This study was supported by the National Key R\&D Precision Medicine Program (2017yfc0908100 to QX) and Shanghai Key Clinical Specialty Grant (Shslczdzk05801 to QX).

\section{Footnote}

Reporting Checklist: The authors have completed the MDAR checklist. Available at http://dx.doi.org/10.21037/ atm-21-92

Data Sharing Statement: Available at http://dx.doi. 
org/10.21037/atm-21-92

Conflicts of Interest: All authors have completed the ICMJE uniform disclosure form (available at http://dx.doi. org/10.21037/atm-21-92). The authors have no conflicts of interest to declare.

Ethical Statement: The authors are accountable for all aspects of the work in ensuring that questions related to the accuracy or integrity of any part of the work are appropriately investigated and resolved. The study was conducted in accordance with the Declaration of Helsinki (as revised in 2013). The protocol was reviewed and approved by the institutional review committee of Renji Hospital (approval No: 2018-136) and individual consent for this retrospective analysis was waived.

Open Access Statement: This is an Open Access article distributed in accordance with the Creative Commons Attribution-NonCommercial-NoDerivs 4.0 International License (CC BY-NC-ND 4.0), which permits the noncommercial replication and distribution of the article with the strict proviso that no changes or edits are made and the original work is properly cited (including links to both the formal publication through the relevant DOI and the license). See: https://creativecommons.org/licenses/by-nc-nd/4.0/.

\section{References}

1. Feltracco P, Barbieri S, Furnari M, et al. Central nervous system infectious complications early after liver transplantation. Transplant Proc 2010;42:1216-22.

2. Singh N, Limaye AP, Forrest G, et al. Combination of voriconazole and caspofungin as primary therapy for invasive aspergillosis in solid organ transplant recipients: a prospective, multicenter, observational study. Transplantation 2006;81:320-6.

3. Husain S, Silveira FP, Azie N, et al. Epidemiological features of invasive mold infections among solid organ transplant recipients: PATH Alliance registry analysis. Med Mycol 2017;55:269-77.

4. Patel MS, Wright AJ, Kohn R, et al. Successful long-term management of invasive cerebral fungal infection following liver transplantation. Mycoses 2015;58:181-6.

5. Wilson MR, Suan D, Duggins A, et al. A novel cause of chronic viral meningoencephalitis: Cache Valley virus. Ann Neurol 2017;82:105-14.

6. Yao M, Zhou J, Zhu Y, et al. Detection of Listeria monocytogenes in CSF from Three Patients with Meningoencephalitis by Next-Generation Sequencing. J Clin Neurol 2016;12:446-51.

7. Mai NTH, Phu NH, Nhu LNT, et al. Central Nervous System Infection Diagnosis by Next-Generation Sequencing: A Glimpse Into the Future? Open Forum Infect Dis 2017;4:ofx046.

8. Jeon YJ, Zhou Y, Li Y, et al. The feasibility study of non-invasive fetal trisomy 18 and 21 detection with semiconductor sequencing platform. PLoS One 2014;9:e110240.

9. Li H, Durbin R. Fast and accurate short read alignment with Burrows-Wheeler transform. Bioinformatics 2009;25:1754-60.

10. Fishman JA, Issa NC. Infection in organ transplantation: risk factors and evolving patterns of infection. Infect Dis Clin North Am 2010;24:273-83.

11. Giovane RA, Lavender PD. Central Nervous System Infections. Prim Care 2018;45:505-18.

12. Pappas PG, Alexander BD, Andes DR, et al. Invasive fungal infections among organ transplant recipients: results of the Transplant-Associated Infection Surveillance Network (TRANSNET). Clin Infect Dis 2010;50:1101-11.

13. Neofytos D, Shoham S, Dierberg K, et al. Diagnostic and therapeutic challenges in a liver transplant recipient with central nervous system invasive aspergillosis. Diagn Microbiol Infect Dis 2012;73:374-5.

14. Saxena S, Gee J, Klieger S, et al. Invasive Fungal Disease in Pediatric Solid Organ Transplant Recipients. J Pediatric Infect Dis Soc 2018;7:219-25.

15. Agnihotri SP. Central Nervous System Opportunistic Infections. Semin Neurol 2019;39:383-90.

16. Pruitt AA. Central Nervous System Infections Complicating Immunosuppression and Transplantation. Continuum (Minneap Minn) 2018;24:1370-96.

17. Lin SJ, Schranz J, Teutsch SM. Aspergillosis case-fatality rate: systematic review of the literature. Clin Infect Dis 2001;32:358-66.

18. Wijdicks EF, Plevak DJ, Wiesner RH, et al. Causes and outcome of seizures in liver transplant recipients. Neurology 1996;47:1523-5.

19. Wilson MR, Naccache SN, Samayoa E, et al. Actionable diagnosis of neuroleptospirosis by next-generation sequencing. N Engl J Med 2014;370:2408-17.

20. Guan H, Shen A, Lv X, et al. Detection of virus in CSF from the cases with meningoencephalitis by nextgeneration sequencing. J Neurovirol 2016;22:240-5. 
21. Fan S, Ren H, Wei Y, et al. Next-generation sequencing of the cerebrospinal fluid in the diagnosis of neurobrucellosis. Int J Infect Dis 2018;67:20-4.

22. Balajee SA, Kano R, Baddley JW, et al. Molecular identification of Aspergillus species collected for the Transplant-Associated Infection Surveillance Network. J Clin Microbiol 2009;47:3138-41.

23. Alastruey-Izquierdo A, Mellado E, Peláez T, et al. Population-based survey of filamentous fungi and antifungal resistance in Spain (FILPOP Study). Antimicrob Agents Chemother 2013;57:3380-7.

24. Negri CE, Gonçalves SS, Xafranski H, et al. Cryptic and rare Aspergillus species in Brazil: prevalence in clinical samples and in vitro susceptibility to triazoles. J Clin Microbiol 2014;52:3633-40.

25. Castro C, Galán-Sanchez F, Linares MJ, et al. A prospective survey of Aspergillus spp. in respiratory tract samples: Species identification and susceptibility patterns.

Cite this article as: Chen F, Zhao Y, Shen C, Han L, Chen X, Zhang J, Xia Q, Qian Y. Next generation sequencing for diagnosis of central nervous system aspergillosis in liver transplant recipients. Ann Transl Med 2021;9(13):1071. doi: 10.21037/atm-21-92
Med Mycol 2019;57:412-20.

26. Dickins B, Rebolledo-Jaramillo B, Su MS, et al. Controlling for contamination in re-sequencing studies with a reproducible web-based phylogenetic approach. Biotechniques 2014;56:134-41.

27. Lusk RW. Diverse and widespread contamination evident in the unmapped depths of high throughput sequencing data. PLoS One 2014;9:e110808.

28. Bukowska OkI, Perlejewski K, Nakamura S, et al. Sensitivity of Next-Generation Sequencing Metagenomic Analysis for Detection of RNA and DNA Viruses in Cerebrospinal Fluid: The Confounding Effect of Background Contamination. Adv Exp Med Biol 2016. [Epub ahead of print]. doi: 10.1007/5584_2016_42.

29. Mongkolrattanothai K, Naccache SN, Bender JM, et al. Neurobrucellosis: Unexpected Answer From Metagenomic Next-Generation Sequencing. J Pediatric Infect Dis Soc 2017;6:393-8. 